\title{
Ciencia y técnica española del I.E.T.c.c. en Iberoamérica*
}

Prof. Dr. JOSE CALLEJA, Vicedirector del IETcc

\section{INTRODUCCION}

Por razones obvias de parentesco y afinidades, de pasado y tradición, siempre ha sido Iberoamérica en general, e Hispanoamérica más en particular, una de las áreas mundiales objeto de predilección por parte de España. Y de modo específico en lo cultural. Dentro de esta parcela, lo científico y lo técnico ha ido cobrando cada vez mayor interés y auge, por exigencias de los tiempos en que vivimos.

En esta proyección cultural científico-técnica de España hacia Iberoamérica, viene destacando en los últimos tiempos la acción del IETCC, dentro del campo del cemento, así como de la construcción en general. Acción que es de doble vertiente: desarrollo de Cursos (los "CEMCO") en España, y Cátedras (la "Eduardo Torroja") en Hispanoamérica, así como la celebración de conferencias, coloquios y mesas redondas por parte del personal más calificado del IETCC, sobre temas de tecnología, calidad y normas, en los mencionados campos.

Precisamente este año se ha desarrollado en "Costillares", con completo éxito, el sexto Curso CEMCO (ya concluido) sobre Patología de la Construcción y de sus Materiales. $\mathrm{Y}$ precisamente también este año han tenido lugar, entre otras, unas actuaciones en Brasil y Argentina por parte del que esto expone. En el terreno de la normativa sobre cementos, en el del empleo de cementos puzolánicos, así como en los aspectos peculiares que presenta la investigación científica y técnica en general, el autor de este trabajo ha dado dos conferencias en Brasil y tres en Argentina, habiendo celebrado en ambos países cuatro largos coloquios en amplias mesas redondas.

\section{ANTECEDENTES}

La Asociación Brasileña de Cemento Portland (ABCP) decidió inaugurar su nueva sede, el Centro Técnico del Cemento situado en la Ciudad Uiversitaria de San Pablo, Brasil, entre los días 24 y 28 de mayo de 1976.

Con tal motivo organizó un Simposio Técnico para conmemorar la inauguración, al cual fueron invitados varios técnicos extranjeros, especialistas en algunos aspectos de la tecnología y de la investigación en el campo del cemento.

* Véase «Presencia y Actividad Cementera Española en Iberoamérica». CEMENTO-HORMICON, núm. 447, págs. 461-466, junio 1971. 
Por parte del Profesor Francisco de Assis BASILIO, antiguo superintendente de la Asociación y actual Presidente del Consejo Técnico de la misma, le fue cursada una invitación verbal previa al Prof. Dr. José CALLEJA, Vicedirector del IETCC, para tomar parte en el acto inaugural del nuevo Centro Técnico y en el Simposio conmemorativo. Invitación que, con aprobación de la Dirección de la Asociación Brasileña del Cemento Portland, fue ratificada por carta del propio Prof. BASILIO de 23 de febrero de 1976.

Al ser aceptada con todo agrado, el que esto escribe se comprometió a pronunciar en el Simposio dos conferencias: una sobre las "Nuevas Normas Españolas para Cementos", dada en el Centro Técnico objeto de la inauguración, ante un auditorio de técnicos de la industria brasileña del cemento con motivo de una de sus reuniones semestrales periódicas -la XXIII-; y otra, dentro del capítulo "Tecnología de los Cementos", sobre "Cemento Portland Puzolánico", dada ante un gran auditorio de técnicos de la industria, de investigadores, de directores de laboratorios y de profesores y alumnos de facultades de ciencias e ingeniería, en el Anfiteatro de la División de Mecánica, del Instituto de Pesquisas Tecnológicas (IPT), de la Ciudad Universitaria de San Pablo.

Ambos temas, aceptados a propuesta de la Asociación, surgieron del interés de ésta y de la industria brasileña del cemento, por conocer más a fondo las particularidades sobre la caracterización de las puzolanas y de su actividad, sobre las correspondientes normas y métodos de ensayo, sobre el mecanismo de la hidratación del cemento bajo acción puzolánica, etc. Asimismo, la exposición acerca de las nuevas normas españolas para cementos sirvió de base para discutir sobre normas para cemento en general, y sobre aspectos particulares de las mismas.

Entre los invitados extranjeros al Simposio figuraban también el Dr. Julio PEREZ ALONSO, Jefe del Laboratorio Central de la Compañía ASLAND; el Ing. Gordon RAY, Director de la Portland Cement Association de los Estados Unidos; y el Dr. Dante J. E. VERONELLI, Asesor Químico de la Corporación Cementera Argentina (CORCEMAR). Por el lado brasileño tomaron parte, además del propio Prof. BASILIO, el Ing. Químico Antonio KROPF SOARES, el Dr. Hans BUCHER, ambos de la ABCP, y el Sr. Carlos R. V. CRUZ, de IBAR.

Al Dr. VERONELLI y al autor de esta exposición les une desde antiguo una fraternal y bien fundamentada amistad, que se inició por contactos profesionales a través de la revista española CEMENTO-HORMIGON, hace ya algunos años. Por esta circunstancia, que no por méritos del que escribe, el Dr. VERONELLI aprovechó la ocasión del Simposio de San Pablo para proponer a los organismos competentes argentinos la actuación de aquél en Buenos Aires. Actuación que podría consistir en tratar los mismos temas que en San Pablo, por ser también de interés para Argentina, así como en celebrar reuniones y mesas redondas con profesionales especialistas en la tecnología del hormigón.

La propuesta del Dr. VERONELLI se plasmó en una invitación formal hecha en carta del Instituto de Tecnología Industrial (INTI), de la Secretaría de Estado de Desarrollo Industrial, firmada por el Interventor del mismo, Mayor Mario A. REMETIN. Esta invitación fue gustosamente aceptada por el que escribe.

\section{PROGRAMA DE LA REUNION DE TECNICOS DE LA INDUSTRIA DEL CEMENTO DEL BRASIL}

Se celebró dicha reunión los días 26 y 27 de mayo de 1976 en el Centro Técnico del Cemento, Ciudad Universitaria de San Pablo, con arreglo al siguiente programa: 


\section{Día 26 de mayo, miércoles}

8.00 a 8.30: Prof. Francisco de Assis BASILIO.

- Apertura de la reunión.

- Asociación Brasileña de Normas Técnicas (ABTN):

"Normas aprobadas, en estudio y grupos de trabajo".

8.30 a 9.30: Ing. Gordon RAY.

- "Actividades de Promoción en la Portland Cement Association (PCA), USA".

9.30 a 10.00: Ing. Químico Antonio KROPF SOARES.

— "El método de Fratini y la experiencia de la ABCP".

10.00 a 11.000 : Prof. Dr. José CALLEJA.

- "Las nuevas normas españolas para cementos".

11.00 a 12.00 : Dr. Julio PEREZ ALONSO.

- "Ensayo acelerado del cemento. Directrices actuales".

\section{Día 27 de mayo, jueves}

8.00 a 8.30: Prof. Francisco de Assis BASILIO.

- "Autocontrol integrado de la producción de cemento".

8.30 a 9.15: Dr. Hans BUCHER.

- "Arena normal RILEM. Su producción en San Pablo: uniformidad de resultados de ensayos entre laboratorios".

9.30 a 10.00: Sr. Carlos R. V. CRUZ.

— "Refractarios: su asentamiento con resina epoxi".

10.00 a 11.00: Reunión de Clausura.

- Propuestas de programa para las reuniones próximas. 


\section{PROGRAMA DEL SIMPOSIO DE INAUGURACION DEL CENTRO TECNICO DEL CEMENTO, DE SAN PABLO, BRASIL}

El Simposio tuvo lugar los días 25, 26 y 27 de mayo de 1976 en el Anfiteatro de la División de Mecánica del Instituto de Pesquisas Tecnológicas (IPT), Ciudad Universitaria de San Pablo, de acuerdo con el programa siguiente:

\section{Día 25 de mayo, martes}

13.00 a 14.30: Inscripción de participantes.

14.30 a 16.00 : Apertura del Simposio.

Tema: PAVIMENTOS DE HORMIGON.

1. ${ }^{a}$ Conferencia: Ing. Gordon RAY.

"Progresos en la técnica de proyecto y ejecución de pavimentos de hormigón".

16.00 a 17.00 : Coloquios.

Día 26 de mayo, miércoles

14.30 a 16.00: Tema: TECNOLOGIA DE LOS CEMENTOS.

2. ${ }^{a}$ Conferencia: Prof. Dr. José CALLEJA.

"Cementos Puzolánicos".

16.00 a 17.00: Coloquios.

\section{Día 27 de mayo, jueves}

14.00 a 15.30: Tema: TECNOLOGIA DEL HORMIGON.

3. ${ }^{a}$ Conferencia: Dr. Dante VERONELLI.

"Reacción árido-álcalis".

15.30 a 16.00: Coloquios.

16.00 a 17.30: Sesión solemne de clausura. Discursos de autoridades. Clausura por el Presidente de la $\mathrm{ABCP}$.

18.00:

Cóctel.

Naturalmente, se giró una visita detallada a la construcción, laboratorios e instalaciones, equipos y aparatos del nuevo Centro Técnico del Cemento. 


\section{ACTUACION ESPAÑOLA EN BRASIL}

\subsection{Del Dr. Julio PEREZ ALONSo}

\subsubsection{En cuanto al tema "Ensayo Acelerado del Cemento: Directrices actuales"}

Al tratar de este tema, el Dr. PEREZ ALONSO expuso una panorámica general del mismo, haciendo destacar su interés dentro del marco del control de calidad de la producción en las fábricas. Describió los fundamentos del procedimiento general y detalló lo más saliente de los métodos más usuales y dignos de confianza, para la puesta en práctica del mismo, en cuanto a la fidelidad y representatividad de sus resultados, en relación con lo que se pretende de ellos: predecir o determinar con antelación suficiente la resistencia normal de un cemento a los 28 días, según una norma dada y unos métodos de ensayo especificados en ella.

A tal efecto explicó con detalle la metodología seguida en el Laboratorio Central de la Compañía ASLAND, a base del tratamiento térmico de probetas de mortero normal de $4 \times 4 \times 16$, ISO-RILEM-CEMBURE.AU. Describió asimismo el equipo ideado y realizado para el tratamiento, y expuso una amplia serie de resultados obtenidos, llegando a la conclusión de que el método sirve para control rutinario de la resistencia de un cemento, con predicción en corto plazo de la normal de 28 días, siempre que, una vez ajustado el método a las condiciones particulares de cada caso -crudo, cocción, enfriamiento, molienda y adiciones- estas condiciones se mantengan sin variación sensible. Es decir, cuando exista prácticamente una constancia de materiales y procesos. Es válido, pues, para el control de una línea de producción dentro de una fábrica.

La exposición del Dr. PEREZ ALONSO dio lugar a un amplio coloquio en el que se suscitaron cuestiones diversas relacionadas con el tema, a las que el ponente respondió con precisión.

\subsection{Del Prof. Dr. José CALLEJA}

\subsubsection{En cuanto al tema "Las Nuevas Normas Españolas para Cemento"}

Lo desarrolló el autor con arreglo al siguiente índice: INTRODUCCION - HISTORIA ACTUALIDAD - FUTURO.

Dijo en la INTRODUCCION que el título general del tema, así como la parte del mismo dedicada en particular a los cementos portland, no implicaba, sólo ni tanto, hablar de las nuevas normas españolas, cuanto de tomar la exposición como base y pretexto para comentar e incluso criticar, en coloquio abierto, aspectos más o menos particulares de las normas para cemento en general, a cuyo efecto el expositor se avendría a hacer, si ello era preciso, de "abogado del diablo".

Ail desarrollar el punto correspondiente a la HISTORIA de las normas españolas para cemento, partió del cambio del Pliego de 1930 por el de 1960, haciendo destacar las características del PCCH-61, así como las diferencias, con éste, del PCCH-64 surgido como revisión del mismo, en cuanto a tipos y categorías resistentes de los cementos y en cuanto a prescripciones de todo tipo y a métodos de ensayo. 
El conferenciante calificó de lógico y liberalizador, en general, el paso del PCCḦ-61 al PCCH-64, e hizo destacar, comentándolas ampliamente, preocupaciones de la época, a su juicio no siempre muy fundadas, sobre la expansión, la retracción y la finura de los cementos.

Trató a continuación del nuevo Pliego RC-75 y de sus diferencias con el PCCH-64, en cuanto a definiciones y denominaciones de los cementos, a los cementos en sí, a las prescripciones o especificaciones y a los métodos de ensayo, llegando a la conclusión de que en determinados aspectos el Pliego RC-75 es ligeramente más liberal que el PCCH-64, y. en otros menos. En todo caso el expositor comentó con detalle tales aspectos.

Al tratar del punto concerniente a la ACTUALIDAD de las normas españolas para cemento, mostró en cuadros generales las definiciones, clasificación, denominaciones y designaciones de los cementos, así como las prescripciones relativas a las características químicas, físicas y mecánicas de los mismos, y las relativas a los que comportan propiedades llamadas adicionales, según el nuevo pliego RC-75.

Con tal motivo hizo una serie detallada de comentarios críticos sobre los más diversos aspectos, tales como tipos, clases y categorías resistentes de los cementos; ensayos de expansión; criterios y ensayos de puzolanicidad; adiciones y "componentes secundarios"; características de los cementos "con propiedades adicionales": blancos, resistentes al yeso y. de bajo calor de hidratación; especificaciones de naturaleza química, etc.

En cuanto al punto relativo al FUTURO de las normas españolas para cemento, el disertante se refirió, por una parte, a la existencia de una Comisión Permanente para el estudio y actualización de las mismas, así como a las orientaciones y tendencias marcadas ya o por marcar por parte de organismos internacionales tales como la ISO, la RILEM, el CEMBUREAU, y más recientemente el CEN: Comité Europeo de Normalización. En este sentido se refirió más concretamente al aspecto de los ensayos de los cementos (en pasta y mortero), y a constancia de relación agua/cemento o a constancia de consistencia.

Concluyó exponiendo que, para complementar el texto oficial del nuevo Pliego RC-75, y con objeto de facilitar la elección de los cementos contenidos en el mismo para su empleo con fines bien determinados, indicando los campos específicos de aplicación de cada tipo, clase y categoría de cemento, el IETCC se había propuesto, como de costumbre en anteriores situaciones de cambio de normas, la redacción de un manual con el carácter de "Código Práctico para el Empleo de los Cementos del Pliego RC-75". Este manual —dijo- reflejará las opiniones y criterios del IETCC al respecto.

Por razones de tiempo, el coloquio previsto al final de la conferencia del Prof. CALLEJA se aplazó para acumularlo al de la conferencia sobre "Cementos Puzolánicos", dada en la tarde del mismo día.

\subsubsection{En cuanto al tema "Cementos Puzolánicos"}

El conferenciante lo desarrolló de acuerdo con el índice que se detalla a continuación:

\section{INTRODUCCION}

1.1. Cuestiones de Nomenclatura

1.2. Justificación de los Cementos Puzolánicos

\section{INSUFICIENCIA DE LOS CEMENTOS PORTLAND}

2.1. Aspectos positivos y negativos de los Cementos Portland

2.2. Remedios para los aspectos negativos 


\section{LAS PUZOLANAS}

\section{LOS CEMENTOS PUZOLANICOS}

4.1. Resistencias mecánicas

4.2. Estabilidad

4.3. Durabilidad

4.4. Calor de hidratación

4.5. Efectos adicionales de la acción puzolánica

\section{COMPONENTES DE LOS CEMENTOS PUZOLANICOS}

\section{CONCLUSION}

En la INTRODUCCION aludió el expositor a la vieja cuestión de si los cementos puzolánicos - como los siderúrgicos- deben o no llevar el sobrenombre o apellido de portland. Para esta cuestión dio a título personal, una solución que justificó.

En cuanto a la otra justificación, es decir, a la razón de ser de los cementos puzolánicos, aportó no una, sino tres: de orden técnico, de orden económico y de orden ecológico, respectivamente. Expuso el punto de vista de la Administración española en cuanto a los aspectos técnico-económicos relativos al empleo y aprovechamiento de materiales puzolánicos, manifestado explícitamente a través del nuevo Pliego RC-75, de cementos.

En el punto 2 de su exposición, el conferenciante hizo resaltar los posibles inconvenientes de los cementos de alta resistencia a toda edad, y en especial a corto plazo, desde el punto de vista de la estabilidad y de la durabilidad. Y a continuación pasó a disertar sobre remedios para tales inconvenientes, entre los cuales, uno de los más eficaces, y, en general, técnica y económicamente viable, es el empleo de materiales puzolánicos. Seguidamente aclaró puntos oscuros sobre fabricación, composición, resistencias y normas, relativas a cementos puzolánicos.

En cuanto al punto 3 relativo a las puzolanas, el expositor quiso referirse específicamente a los criterios y métodos para la evaluación de la acción y del efecto puzolánico.

Enlazó el desarrollo de este punto con el del 4 siguiente, sobre cementos puzolánicos, al tratar de la normalización de éstos y de la necesidad, dentro de ella, de análogos criterios y procedimientos de valoración de la puzolanicidad de los cementos llamados puzolánicos. Dio y justificó su opinión personal al respecto, en el sentido de abogar por el ensayo de cada cemento puzolánico, como un todo indivisible, en los aspectos de resistencias mecánicas y de puzolanicidad (ensayo de FRATINI), complementados con los de estabilidad (agujas LE CHATELIER), durabilidad y calor de hidratación. En cuanto a efectos adicionales de la acción puzolánica en los morteros y hormigones frescos, se refirió a la plasticidad y trabajabilidad de los mismos y a su idoneidad para los tratamientos térmi$\cos$ de endurecimiento acelerado. $\mathrm{Y}$ respecto de otros efectos sobre morteros y hormigones endurecidos, mencionó los relacionados con la reacción árido (reactivo)-álcalis (del cemento) y a la impermeabilidad, flexibilidad, elasticidad y capacidad de fluencia de los hormigones puzolánicos.

Finalmente, en el punto 5 relativo a los componentes de los cementos puzolánicos, el conferenciante mencionó las características más idóneas de aquéllos para que éstos resulten óptimos. En la conclusión justificó el enfoque deliberadamente dado a su exposición.

Al final de la conferencia tuvo lugar un intenso y extenso coloquio, en el que participatron bastantes de los numerosos asistentes. Se le formularon al conferenciante del orden de 25 preguntas, a las cuales respondió empleando en ello unos 90 minutos. Las cuestio- 
nes propuestas y contestadas versaron tanto sobre el tema de los cementos puzolánicos, como sobre el de las normas para cemento, y se refirieron principalmente a:

i) categorías resistentes de los cementos según sus tipos y clases, dispersión de los resultados de los ensayos de resistencias y causas de la misma (heterogeneidades de composición, finura, granulometría, etc.);

ii) especificaciones de tipo químico en las normas, particularmente en lo referente al residuo insoluble y al trióxido de azufre de los cementos puzolánicos;

iii) interpretación, valor y fiabilidad de los ensayos de expansión en autoclave, dispersión de los resultados de los mismos en el caso de los cementos portland y puzolánicos, y causas;

iv) interpretación, valor y fiabilidad de los resultados del ensayo de puzolanicidad por el método de FRATINI;

v) finuras y granulometrías de cementos portland y puzolánicos con puzolanas naturales y cenizas volantes. Molienda por separado de clínker y puzolanas;

vi) productos de hidratación (tobermoríticos) en los cementos portland y puzolánicos. "Efecto silícico" en estos últimos;

vii) curado térmico acelerado y temperaturas máximas y óptimas de los tratamientos correspondientes;

viii) proporciones óptimas de mezclas de clínkeres y puzolanas de todo tipo;

ix) empleo de kieselguhr y tierras de diatomeas e infusorios en la fabricación de cementos puzolánicos;

x) absorción de agua y retracción química, hidráulica y térmica de cementos puzolánicos en general, y en particular con cenizas volantes;

xi) empleo de aditivos en general, y de aceleradores en particular, con cementos de tipo diverso;

xii) efectos de la posible aplicación de cementos puzolánicos y siderúrgicos en inyecciones de vainas de pretensado;

xiii) dosificación de yeso en los cementos puzolánicos;

xiv) determinación de granulometrías parciales por tamizado en húmedo y otros métodos.

Por su relación parcial con el tema de "Cementos Puzolánicos" se hace mención aquí de la conferencia, extraordinariamente interesante, dada por el Dr. Dante VERONELLI acerca de la reacción entre áridos reactivos y álcalis de los cementos, que constituyó una verdadera y completa puesta al día del tema, de una forma clara, ordenada y sistemática.

En ella se hizo destacar los mecanismos de la reacción, a base de la formación de silicatos alcalinos hidratados coloidales, entre la sílice activa del árido y los álcalis disponibles del cemento. Estos silicatos constituyen películas permeables, a través de las cuales, en presencia de agua, se producen fenómenos de naturaleza osmótica y de carácter expansivo y disruptor.

Señaló también que este tipo de reacciones expansivas se atenuaban o inhibían con la presencia de adiciones muy activas en el cemento, ricas en sílice y menos ricas en alúmina, del tipo de las puzolanas, poniendo el ejemplo dado por el Dr. IDORN, especialista en el tema de la reacción árido-álcalis, de inhibición puzolánica producida por la acción del "moller" mezclado con el cemento, en presencia de áridos reactivos en Islandia.

Las actividades del Simposio tuvieron amplio eco en la prensa brasileña de San Pablo. 


\subsubsection{Visita al Centro de Control Tecnológico del Hormigón, del Profesor BAUER}

Digna de mención, dado el volumen e importancia de este Centro, es la visita realizada al mismo, por amable invitación de sus promotores y directivos, el Prof. L. A. FALÇAO BAUER, Ingeniero Civil, y su eficaz y activa colaboradora María A. A. NORONHA.

Se trata de un centro privado que tiene las dimensiones de un verdadero Instituto de investigación, control y ensayo de materiales, y que de hecho lleva a cabo los tres cometidos, además del de proyectar y diseñar.

A tenor de ello, su espacio, edificios, instalaciones y equipos de material y de personal -éste último por lo que respecta a su número, profesionalidad y competencia-, son los que cabe esperar para una labor eficaz y provechosa, tanto para el Centro, como para los usuarios del mismo y para el país. Y la prueba de esa eficacia está en el hecho de que la institución, como privada que es, no recibe aportación oficial alguna, no obstante lo cual se autofinancia y rinde beneficios.

Pero los primeros y más directos beneficiarios son las empresas constructoras que, a través del Centro, pueden llevar a cabo un riguroso control de los materiales que utilizan, así como de los elementos y estructuras que construyen. En este aspecto se realizan numerosos ensayos de cementos, áridos, aceros para armar y hormigones.

Una de las facetas que ponen de relieve la amplitud de miras con que los servicios del Centro están concebidos, así como la diligencia y eficacia con que son prestados a los usuarios, es la disponibilidad permanente de equipos para el ensayo de aceros en el acto. De tal manera que el camión que entra en el Centro con un cargamento de redondos para armar, sale del mismo con el certificado en el que se dan las resistencias recién obtenidas en los ensayos inmediatamente efectuados.

Por este y otros detalles no es exagerado afirmar que el Centro del Ing. BAUER constituye una organización, que por su planteamiento, realización y esquema operativo puede servir como modelo de utilidad y eficacia.

\section{ACtuacion española del PROF. DR. JOSE CALLeJa EN ARgentina}

\subsection{En general}

Esta actuación se anunció en la Sección de ARQUITECTURA, INGENIERIA, PLANEAMIENTO Y DISENO, en la página 20 del diario CLARIN de Buenos Aires, del Viernes 21 de mayo de 1976, con el título: "Nos visitará el Vicedirector del Instituto Eduardo Torroja".

En el texto, comunicado por el Instituto del Cemento Portland Argentino (ICPA) se daba cuenta de la visita, por invitación del Instituto Nacional de Tecnología Industrial (INTI).

Asimismo se anunciaban las dos conferencias que el invitado daría en el salón de actos de la Sociedad Científica Argentina (equivalente a la Real Academia Española de Ciencias Exactas, Físicas y Naturales), y se incluía un breve "curriculum vitae" del Prof. Dr. J. CALLEJA. 


\subsection{Programa}

Se desarrolló con arreglo al siguiente plan, ampliado en relación con el original que comprendía sólo las dos conferencias mencionadas:

\section{Día 31 de mayo, lunes}

A las 15.00 horas, en la sede del INTI:

- Mesa redonda sobre el tema: HORMIGON EN GRANDES MASAS.

\section{Día 1 de junio, martes}

A las 11.00 horas:

- Visita a CORCEMAR, S.A.: Sres. J. B. y R. D. VERZINI (Corporación Cementera Argentina).

A las 18.30 horas, en la sede de la Sociedad Científica Argentina:

- Conferencia sobre: CEMENTOS PUZOLANICOS.

- Coloquio.

\section{Día 2 de junio, miércoles}

A las 9.00 horas:

- Visita al Parque Tecnológico MIGUELETE, del INTI.

- Coloquio sobre Metrología.

A las 18.30 horas en la sede de la Sociedad Científica Argentina:

- Conferencia sobre: CRITERIOS PARA LA NORMALIZACION DEL CEMENTO PORTLAND.

- Coloquio.

\section{Día 3 de junio, jueves}

A las 10.30 horas, en la sede de la Sociedad Científica Argentina:

- Conferencia sobre: CONSIDERACIONES GENERALES APOLOGETICAS ACERCA DE LA INVESTIGACION CIENTIFICA Y TECNICA. 


\subsection{Mesa redonda sobre "HORMIGON EN GRANDES MASAS"}

Se prolongó por espacio de unas cuatro horas y se desarrolló con arreglo al siguiente temario previsto:

I) Especificación de Cementos.

II) Control de Cementos.

III) Generación de Calor en la Hidratación.

IV) Empleo de Puzolanas y otros Aditivos.

V) Reacción Arido-Alcalis.

VI) Criterios actuales para la Selección de Materiales.

En realidad se trataron muchos otros temas colaterales, surgidos o planteados alrededor o al margen del temario.

La mesa redonda tuvo lugar con la participación de numeroso público científico y técnico interesado en las materias debatidas, ante un panel constituido por los siguientes participantes, enunciados por orden alfabético según programa:

1. Ing. Héctor J. BUNGE, del INTI.

2. Ing. Luis M. CALVO, de la Comisión Técnica Mixta Salto Grande.

3. Dr. Alsiades CATALANO, del INTI.

4. Ing. José COLINA, de la Asociación Argentina de Tecnología del Hormigón.

5. Ing. Alberto FAVA, de la Facultad de Ingeniería de la Universidad La Plata.

6. Ing. Alberto GIOVAMBATTISTA, de la Asociación Argentina de Tecnología del Hormigón.

7. Ing. Alberto L. GRANDI, de la Facultad de Ingeniería de la Universidad de Buenos Aires.

8. Ing. Mario ORLANDO, del Organismo Agua y Energía.

9. Ing. Manuel SABESINSKI FELPERIN, del Instituto de Mecánica Aplicada a las Estructuras, de la Universidad de Rosario.

10. Dr. Dante J. E. VERONELLI, del Instituto del Cemento Portland Argentino.

11. Un representante del Ente Binacional Yaciretá Apipé.

Al informador le parece, no obstante, que en el panel formaron parte algunas personas más de las enunciadas, las cuales siente no recordar en su totalidad.

El Prof. Dr. José CALLEJA figuraba en este panel en calidad de invitado especial, siendo presentado al auditorio por el Ing. Héctor J. BUNGE que lo presidía.

\subsubsection{Introducción}

Hecha la presentación y expuesto el propósito de la mesa redonda el Ing. BUNGE concedió la palabra al Prof. CALLEJA. 
Tras de agradecer éste al INTI en las personas del Mayor REMETIN y del Dr. CATALANO y al ICPA en las del Ing. DUVOY y del Dr. VERONELLI la invitación recibida, comenzó haciendo una introducción general a cada uno de los seis puntos del temario, con objeto de presentar materia de partida, base para el coloquio.

Muy en breve, esta introducción fue como sigue:

- En cuanto al título general HORMIGON EN GRANDES MASAS, señaló que el campo de aplicación de este material se podía circunscribir, por fijar ideas, a las presas y a las cimentaciones masivas -eventualmente se puede hacer extensivo dicho campo al caso de determinadas obras marítimas: diques, muelles, escolleras, etc.- .

- Delimitado así el ámbito, pasó a glosar el primer punto sobre ESPECIFICACIONES DE LOS CEMENTOS en cada uno de los dos sectores señalados.

En el de las presas indicó que eran dos las características principales que exigía este tipo de obras: Resistencia (mecánica para resistir el empuje del agua, y química para resistir la posible agresión -ácida mineral, carbónica o salina- de distintas aguas según los casos), y Capacidad de retención.

En cuanto a la resistencia mecánica, el Prof. CALLEJA señaló que en ningún caso debía constituir un problema, pues, a tal efecto, cualquier tipo de cemento suficientemente resistente podía ser utilizado en principio, con tal de que las condiciones de proyecto, cálculo, otros materiales, dosificación, ejecución, puesta en obra, compactación, curado y control fuesen cumplidamente las adecuadas.

En cuanto a la resistencia química, hizo resaltar que la cuestión era distinta; ya que podrían surgir problemas, puesto que las aguas puras, carbónicas agresivas y àcidas, con gran capacidad de disolución y ataque, eran susceptibles de provocar un "deslavado" de la cal de hidrólisis del cemento, dando lugar a un aumento de porosidad y a un debilitamiento del hormigón. Y habida cuenta también de que las aguas salinas, particularmente las sulfatadas, eran susceptibles de formar yeso con dicha cal de hidrólisis y ettringita con los aluminatos del cemento, dando pie a reacciones expansivas destructoras del hormigón. En tales casos, no todos los cementos son igualmente aptos.

Para mejor resistir los ataques de tipo ácido señaló como más idóneos los cementos puzolánicos, ya que fijan la cal de hidrólisis de forma estable; y para aguantar mejor la agresión salina de los sulfatos indicó los cementos de bajo contenido de aluminato tricálcico ( $\mathrm{y}$ de baja suma de aluminatos más ferritos) dentro de los portland, los propios cementos puzolánicos $\mathrm{y}$, cómo no, los cementos siderúrgicos.

Por lo que respecta a la capacidad de retención de agua por parte de una presa, el Prof. CALLEJA apuntó la ausencia de grietas y fisuras en el paramento de la misma, como condición necesaria. Para ello citó como más idóneos los cementos de baja retracción, tanto química (irreversible) como hidráulica y térmica (reversibles -al menos en parte-). A tal efecto indicó como aptos los cementos de bajos contenidos de aluminato tricálcico y de álcalis, de baja finura y de bajo calor de hidratación. En consecuencia, los cementos portland resistentes al yeso y a los sulfatos en general y, sobre todo, los cementos puzolánicos que fijan cal dando geles tobermoríticos más silícicos (de menor relación cal/sílice), los cuales por su acción colmatante, rellenan y taponan poros e impermeabilizan en una cierta medida el hormigón (reducen la penetrabilidad del agua y de las disoluciones acuosas).

En resumen, de lo anterior sacó el Prof. CALLEJA la conclusión de que, para presas, los cementos más idóneos serían los puzolánicos iconstituidos por un 30-40\% de una puzolana muy activa y por un 70-60\% de un clínker con menos de $5 \%$ de aluminato tricálcico*

* Tanto menor aluminato tricálcico en el clínker cuanto mayor contenido de alúmina en la puzolana. 
y menos de $22 \%$ de suma de aluminato tricálcico y ferrito aluminato tetracálcico, que fuesen al mismo tiempo de bajo calor de hidratación (menos de $65 \mathrm{cal} / \mathrm{g}$ a 7 días y menos de $75 \mathrm{cal} / \mathrm{g}$ a 28 días), datos numéricos dados a título orientativo y que, por fijar ideas, corresponden a prescripciones de las nuevas normas españolas para cemento.

En el campo de las cimentaciones el expositor señaló que las principales características que requería este tipo de obras eran: Resistencia (mecánica) y química frente a aguas, y suelos agresivos, y Estabilidad.

En lo que se refiere a la resistencia mecánica es válido — dijo- cuanto queda indicado para el caso de las presas, siendo idóneo, en principio, cualquier tipo de cemento. En cuianto a la resistencia química, si por delimitar el problema se consideran posibles ataques por aguas ácidas y por aguas y suelos yesíferos, los cementos más aptos serían por orden de eficacia, los puzolánicos con clínker bajo en aluminatos y ferritos, y los puzolánicos ordinarios, en el primer caso; y los siderúrgicos en una u otra de sus distintas clases, según la naturaleza del medio atacante y el contenido de escoria siderúrgica del cemento. Sin olvidar —advirtió el disertante- los posibles cementos ternarios a base de clínker, escoria y puzolana.

- Al glosar el segundo punto relativo a CONTROL DE CEMENTOS, el Prof. CALLEJA señaló como ensayos adecuados, necesarios y posiblemente suficientes, de acuerdo con lo que precede, los de puzolanicidad — por el método de FRATINI- en el caso de los cementos puzolánicos; los de análisis para determinar la idoneidad química de la escoria, en el caso de los cementos siderúrgicos; los cálculos de la composición potencial o las determinaciones de la composición mineralógica por difracción de rayos $\mathrm{X}$ o por microscopía, en el caso de los cementos portland, más o menos resistentes a yeso y sulfatos; y los de resistencia mecánica, estabilidad - agujas Le Chatelier-y calor de hidratación, en todos los casos.

- Al tratar del punto tercero relativo a la GENERACION DEL CALOR DE HIDRATACION, el ponente puso de relieve que los componentes del cemento que mayor calor liberaban en el proceso de su hidratación eran el aluminato tricálcico -a más corto plazo- y el silicato tricálcico - a más largo plazo-; que el aspecto fundamental idel calor de hidratación liberado era la temperatura que a consecuencia de él tomaba el hormigón -y los consiguientes gradientes térmicos de calentamiento y enfriamiento, con las secuelas de la retracción térmica y de la fisuración y agrietamiento del material-; que la temperatura alcanzada por el hormigón, no sólo dependía del calor de hidratación del cemento (en cuanto a cantidad total desprendida y velocidad de desprendimiento), sino también de la geometría del hormigón (masa, relación superficie/masa, espesores, etc.), y de las condiciones ambientales, así como del empleo de aceleradores del fraguado y/o del endurecimiento - un acelerador no modifica en general, la cantidad total de calor desprendida, pero acelera el desprendimiento-.

Añadió que los métodos experimentales para determinar el calor de hidratación y sus efectos eran, en esencia, el de disolución que determina realmente cantidad de calor, y el adiabático o de la botella aislante (frasco "thermos"), que mide más bien el aumento de temperatura de la masa que fragua en condiciones adiabáticas. Este último parece, en definitiva, el más convincente a efectos comparativos, por ejemplo, en el caso de seleccionar cementos para construir una presa.

- En el punto cuarto relativo al EMPLEO DE PUZOLANAS Y OTROS ADITIVOS, el Prof. CALLEJA dijo, que, simplistamente expuestas, las acciones de las puzolanas eran tres: diluir el cemento, fijar la cal liberada en la hidratación, e incrementar los geles tobermoríticos de la pasta hidratada, dando lugar a productos de relación cal/sílice menor que la normal. Y que, en consecuencia, de estas acciones se deducían los correspondientes efectos, previsibles y comprobados de las puzolanas, en cuanto que: re- 
bajan el calor de hidratación (cantidad total y velocidad de desprendimiento) de las masas que fraguan, reduciendo la temperatura máxima alcanzada por las mismas; aumentan la resistencia química frente a ataques de agentes y medios agresivos; aumentan la impermeabilidad o "impenetrabilidad" del hormigón al agua y a las disoluciones acuosas; disminuyen la retracción.

En cuanto a OTROS ADITIVOS, las cenizas volantes son puzolanas a los efectos señalados —dijo el Prof. CALLEJA-, y las escorias siderúrgicas tienen propiedades hidráulicas latentes o potenciales intrínsecas, las cuales se actualizan y ponen en juego mediante activación por la cal de hidrólisis del cemento, ejerciendo análogas o similares acciones que las puzolanas, con semejantes o parecidos efectos.

- En su comentario al punto quinto concerniente a la REACCION ALCALI-ARIDO el opinante se ciñó al aspecto de su evitación, haciendo destacar el hecho de que uno de los medios más eficaces de conseguirla consistía precisamente en el empleo de puzolanas. $\mathrm{Y}$ esto, con independencia del hecho paradójico de que las puzolanas sean, por definición, materiales reactivos frente a los álcalis del cemento. En efecto -aclaró el Prof. CALLEJA - al estar las puzolanas muy finamente molidas, e íntima y homogéneamente mezcladas con el cemento, y al intervenir en una proporción masiva considerable en relación con éste, dan lugar a una "reacción álcali-puzolana" preferente a la "reacción álcali-árido", y más rápida, completa, generalizada atenuada e inoperante que ella. Con lo cual se resta posibilidad a la reacción localizada álcali-árido, que es la que verdaderamente tiene carácter destructivo. Es el caso clásico de diluir un problema para suavizar sus efectos, en virtud del aforismo "divide y vencerás", o por la sencilla ley física de que lo que se gana en extensión se pierde en intensidad (producto constante de dos magnitudes conjugadas).

- Finalmente, al tratar del punto sexto y último referente a CRITERIOS ACTUALES PARA LA SELECCION DE MATERIALES (para hormigón en grandes masas: presas y/o cimentaciones), el Prof. CALLEJA hizo una especie de resumen de todo la anteriormente expuesto, aludiendo a cada uno de los materiales unitarios que constituyen el hormigón en masa: cementos, áridos, aguas, aditivos (eventualmente), y al propio hormigón como un todo.

En cuanto a los cementos, señaló, en virtud de lo dicho, los puzolánicos, los siderúrgicos y los portland con adiciones activas (o "componentes secundarios"): escorias y/o puzolanas naturales (o artificiales) y cenizas volantes, como los más idóneos.

Con respecto a los áridos hizo destacar la necesidad de que fueran estables (per se: no deleznables; frente a cementos: no susceptibles de reacción expansiva con los álcalis) y no absorbentes.

En relación con las aguas para el amasado del hormigón indicó la conveniencia de que cumplieran los requisitos exigidos en las normas, instrucciones y recomendaciones vigentes, particularmente con el de no contener sustancias que alteren perjudicialmente el fraguado y el endurecimiento normales del cemento.

Respecto de los aditivos, mencionó la posibilidad de utilizar, según los casos y las exigencias o conveniencias en relación con los mismos: retardadores de fraguado -que faciliten el transporte, la puesta en obra y la compactación del hormigón fresco-; reductores de agua ("superplastificantes") que rebajen la relación agua/cemento, sin merma de la plasticidad o trabajabilidad -asentamiento del cono Abrams-; o inclusores de aire que confieran al hormigón, cuando lo precise, una mayor resistencia a las heladas. Hizo observar lo mucho que cabría hablar acerca del uso, del mal uso y del abuso de los aditivos. 
Finalmente, con relación al propio hormigón puso de relieve la necesidad de que fuese lo más compacto e impermeable ("impenetrable") posible, para lo cual no bastaba que todos y cada uno de los materiales reseñados cumpliesen en todos los casos con los máximos requisitos de idoneidad y aptitud, sino que era preciso, antes o simultáneamente, que el proyecto, el cálculo, la dosificación, la ejecución, la puesta en obra, la compactación, el curado y el control de calidad del hormigón fresco y endurecido fuesen en todo momento los adecuados.

\subsubsection{Coloquio}

Al final de esta introducción el Presidente, Ing. BUNGE, abrió un coloquio en el que, tanto por parte de los componentes del panel como por parte de los técnicos presentes se formularon muy diversas preguntas, sencillas y múltiples, no sólo sobre el temario, sino también ajenas al mismo, a las que contestó el Prof. CALLEJA.

En las preguntas y en las respuestas se entremezclaron aspectos que, entre otros, versaron principalmente sobre los siguientes puntos:

i) criterios acerca del empleo de puzolanas naturales y de cenizas volantes añadidas a los cementos en obra;

ii) valor de la superficie específica medida por permeabilidad al aire (BLAINE), como índice de finura, en el caso particular de los cementos puzolánicos;

iii) papel de los finos del cemento en general, y de los puzolánicos en particular, en cuanto a resultados de expansión en autoclave;

iv) papel de la magnesia en sus diversas formas y con distintos contenidos en el cemento, en cuanto a resultados del ensayo de expansión en autoclave;

v) valor e interpretación de las prescripciones de las normas, relativas al contenido máximo tolerable de magnesia en los cementos, con vistas a la expansión de los mismos en autoclave;

vi) crítica y enjuiciamiento de los métodos para medir el calor de hidratación por disolución y por el procedimiento adiabático;

vii) valor práctico de los resultados de uno y otro método en el caso de grandes masas de hormigón;

viii) velocidad de desprendimiento de calor, calor total desprendido en la hidratación del cemento, velocidad de disipación del mismo y temperatura máxima alcanzada por una masa de hormigón, en función de diversas variables y circunstancias;

ix) el factor temperatura en el hormigonado, y hormigonado en tiempo cálido;

x) empleo de cementos tipo IV ASTM en el hormigonado en tiempo cálido;

xi) empleo de hielo en el amasado de hormigón en tiempo cálido y su influencia en la trabajabilidad del hormigón;

xii) ensayos con "hor migón filtrado" para estudios relativos, entre otros aspectos, al de la disipación del calor en función de los cementos, áridos y aditivos eventualmente empleados;

xiii) aplicación de las técnicas de medidas eléctricas para el estudio del fraguado del hormigón en condiciones especiales; 
xiv) reacción árido-álcalis: necesidad de agua para la realización del fenómeno osmótico ligado a ella;

xv) métodos acelerados para la detección y evaluación de la reacción árido-álcalis;

xvi) remedios para atenuar o inhibir dicha reacción: empleo de cementos puzolánicos con clínkeres bajos en álcalis (según las especificaciones de las normas);

xvii) influencia de la concentración de ópalo, calcedonia, etc., en los áridos, en cuanto a los efectos de su reacción con los álcalis del cemento;

xviii) influencia de los modernos sistemas y hornos de clinkerización en la retención y acumulación de álcalis en los cementos;

xix) influencia de áridos basálticos con nontronita, saponita y feldespatos caolinizables, en la estabilidad de los hormigones.

Contestaron y opinaron también sobre algunos de estos puntos los componentes del panel, y el Prof. CALLEJA pidió al Dr. VERONELLI que respondiese en detalle a las preguntas relativas a las cuestiones de la reacción árido-álcalis, en las cuales éste se halla muy impuesto.

\subsection{Conferencia sobre "Cementos Puzolánicos"}

\subsubsection{Contenido}

Fue el mismo que el Prof. CALLEJA expuso en Brasil y que queda reseñado en el punto 4.2 .2 .

\subsubsection{Coloquio}

Se prolongó por un tiempo aproximado de una hora, durante el cual se formularon preguntas y se suscitaron cuestiones, en número de unas 25 , que versaron sobre superficie específica de los cementos puzolánicos; sobre trabajabilidad y retención de agua de los hormigones y morteros confeccionados con ellos; sobre fisuración y resistencia al agua de mar de los hormigones puzolánicos; sobre cementos siderúrgicos y con adiciones activas — componentes secundarios-; etc.

\subsection{Conferencia sobre "Criterios para la Normalización de los Cementos"}

\subsubsection{Contenido}

Fue el mismo expuesto por el Prof. CALLEJA en San Pablo, con el texto que queda reflejado en el punto 4.2.1. 


\subsubsection{Coloquio}

Se desarrolló en un tiempo de unos 45 minutos y en él se plantearon cuestiones en número aproximado de 15 , las cuales trataron acerca de resistencia a distintas edades de las diversas categorías de cementos; de prescripciones de carácter físico, mecánico y químico según las normas; de metodología de ensayos, etc.

\subsection{Conferencia sobre "Consideraciones Generales Apologéticas acerca de la Investigación Científica y Técnica"}

\subsubsection{Contenido}

Conversaciones de carácter general mantenidas con miembros del INTI y del ICPA, sobre el tema de la investigación en el mundo, dentro del campo del cemento, indujeron al Prof. CALLEJA a ofrecer una conferencia suplementaria sobre el tema considerado en general, ofrecimiento que fue acogido con el máximo interés y aceptado.

La conferencia, con el título que queda expuesto, se dio en la sede de la Sociedad Científica Argentina en la que cumplimentaron al Prof. CALLEJA el Secretario de Estado para el Desarrollo Industrial, Dr. PODESTA y el Presidente de la Sociedad.

El auditorio fue numeroso, con la asistencia de las personalidades señaladas y de los miembros directivos y jefes de departamentos y servicios del INTI, del ICPA, así como de diversas universidades, facultades de ingeniería, institutos, asociaciones, comisiones, entidades y centros argentinos de docencia e investigación, ensayo de materiales y tecnología del hormigón, tanto oficiales como privados. La conferencia se desarrolló con arreglo al siguiente índice:

0. Introducción.

1. Panorama actual de la investigación científico-técnica.

2. Defensa de lo virtualmente inútil.

3. Tipos de investigación.

4. Elementos de la investigación.

4.1. El investigador.

4.1.1. La creación.

4.1.2. El espíritu de trabajo.

4.1.3. El espíritu observador y crítico.

4.1.4. La ética del investigador.

4.1.4.1. Misión docente del investigador.

4.2. Los temas y la finalidad.

4.2.1. El planteamiento y el desarrollo.

4.3. Los medios.

4.4. La formación científica en y para la investigación.

5. Conclusión. 
En su exposición, profundamente ilustrada con datos históricos y anecdóticos de relevantes figuras de la ciencia y de la tecnología de todos los tiempos, el Prof. CALLEJA adoptó como lema o divisa la justificación y la apología de la investigación, como misión de todas las épocas, y en particular de la presente, indispensable para la cultura, el progreso y el bienestar de la humanidad.

Hizo hincapié en la necesidad de dar a conocer al hombre medio lo que es la investigación, su necesidad y sus ventajas, así como en la urgencia de aceptar el hecho inconcuso de que no existe conflicto entre teoría y pragmatismo, ciencia pura y ciencia aplicada o tecnología, e investigación básica o fundamental y aplicada, técnica o de desarrollo e innovación. Y esto, porque no hay conocimiento ni saber del que no pueda obtenerse, pronto o tarde, alguna utilidad, pues toda noción es un instrumento en el que radica una energía potencial que es preciso tratar de actualizar, ya que los únicos conocimientos que jamás se aplican son los que no se tienen, ni se deja de ser práctico por poseer una amplia base teórica, sino por no usarla o por usarla mal. Esto quiere decir, según el Prof. CALLEJA, que la Ciencia llamada pura de una época, más o menos tarde sirve de base firme e insustituible a la Ciencia llamada aplicada de épocas posteriores, lo que hace que el avance de la ciencia y. de la técnica sea una especie de carrera de relevos contra el tiempo. Y quiere decir también que si la Ciencia ha surgido de dos exigencias del hombre: la de saber y la de vivir mejor, sólo ha podido satisfacer la segunda después de satisfecha la primera, y precisamente por ello. No otra es la razón de que en gran parte - por no decir en toda- de las pesquisas encaminadas a obtener logros tecnológicos se considera hoy día como útil y necesaria, y a veces imprescindible, una cierta "investigación básica de apoyo".

El Prof. CALLEJA desarrolló los diversos puntos relativos a los distintos elementos que intervienen en la investigación, con especial énfasis en la labor formativa y docente que incumbe a la misma, a fin de "crear escuelas" que aseguren su continuidad, progreso y eficacia, así como en la formación que debe recibir y poseer el investigador.

Al final de su disertación el Prof. CALLEJA concluyó que la investigación científica, cualesquiera que sean sus atributos y finalidades, es empresa rentable de las comunidades $\mathrm{y}$, a la corta o a la larga, fuente de progreso y desarrollo tecnológico, y acelerador del crecimiento, del bienestar y del nivel de vida de los pueblos.

Añadió que, por comprenderlo así, los más perspicaces de entre estos pueblos promueven y fomentan la investigación científica y técnica, dedicando a ambas la atención y los fondos que merecen en la medida en que pueden, para no perder primacía o rango si los tienen, o para adquirirlos si no los poseen.

Terminó diciendo el Prof. CALLEJA que si alguien pudiese llegar a pensar y creer que la investigación es un lujo, habría que pedirle que fuera lo suficientemente ecuánime y conciliador como para admitir sin reservas que se trataría de un lujo barato; mucho más barato, por supuesto, que el lujo carísimo de la falta de investigación, con sus consecuencias obligadas de importación de patentes y licencias, pago de royalties y dependencia tecnológica - verdadero colonialismo industrial- de países más desarrollados y perspicaces. Dependencia que, lejos de reducirse, se incrementaría con el tiempo, pues si un país cree que puede pasar sin investigación, no está en cambio dispuesto a creer que puede prescindir de los adelantos y facilidades de que gozan los países que investigan, naturalmente que como consecuencia de la investigación que llevan a cabo. El rápido desarrollo y el nivel de vida elevado hay que pagarlos, y la forma más barata -o si se prefiere, menos cara- de hacerlo, es desarrollar la investigación para que ésta dé lugar a su vez al desenvolvimiento y a la innovación de la tecnología. 


\subsection{Visita a la Corporación Cementera Argentina (CORCEMAR)}

En la sede de esta Corporación el Prof. CALLEJA fue presentado por el Dr. Dante VERONELLI, Asesor químico de la misma, al Presidente de su Directorio, Don Raul Dante VERZINI - "Don Raul" por antonomasia- y a su hermano el Ing. José Bruno VERZINI, Superintendente de la Planta Mendoza de CORCEMAR a quien el Prof. CALLEJA ya tenía el gusto de conocer desde su anterior paso por Buenos Aires en marzo de 1971.

La entrevista fue grata por demás, dada la personalidad humana de Don Raul. Es éste uno de los principales pioneros de la industrialización y comercialización del cemento portland en Argentina, misión en la que comenzó en 1925 y a la que ha dedicado, hasta el momento, 50 años largos de su ininterrumpida y fecunda actividad. Dentro de ésta, y aparte de la faceta industrial, en la que desde Secretario Técnico de la primitiva fábrica de VERZINI, GARLOT Y CIA. -Cemento "Sigma"- y de CORCEMAR, S. A., pasando por la Superintendencia de las fábricas de Mendoza y Pipinas y por la representación de CORCEMAR en Buenos Aires llegó a Vocal-Director y a Presidente desde 1956, Don Raul ha presidido varias veces la Asociación de Fabricantes de Cemento y el Instituto del Cemento Portland Argentino. Mucho bueno habría que añadir acerca de otros múltiples aspectos humanos de los Sres. VERZINI, quienes como los ya fallecidos Don Alfredo FORTABAT, Presidente que fue de LOMA NEGRA, Compañía Industrial Argentina, S. A. - la de mayor producción del país con cinco fábricas-, y Don Héctor Bartolomé MINETTI, Presidente de la Compañía Sudamericana de Cemento Portland "Juan Minetti e Hijos", Ltda., S. A., con tres fábricas, son los más destacados creadores de la industria cementera argentina y los promotores de su desarrollo.

CORCEMAR es la segunda firma productora de cemento del país, con cuatro fábricas respectivamente situadas - y cronológicamente levantadas- en Córdoba, Mendoza, Pipinas (a $100 \mathrm{~km}$ de La Plata) y. Yocsina.

\subsection{Visita al Parque Tecnológico MIGUELETE, del INTI}

\subsubsection{Visita}

El Parque Tecnológico MIGUELETE es la zona donde el INTI tiene desplegados todos sus medios de acción para llevar a cabo las misiones que le son encomendadas por los más diversos sectores industriales. Bajo la dependencia de la Secretaría de Estado de Desarrollo Industrial, del Ministerio de Economía, presta servicios de asistencia técnica a dichos sectores en problemas de:

i) estudios de idoneidad de materias primas;

ii) estudio de idoneidad y precisión de procesos fabriles;

iii) determinación de causas de fallos de materiales y estructuras;

iv) verificación, calibración y control de instrumental y equipos y aparatos de medida;

v) estudios de corrosión y de recubrimientos metálicos;

vi) estudios de soldadura;

vii estudio de sistemas de envasado. 
Cuenta para ello con una gran variedad de instalaciones, equipos, técnicas y aparatos a cargo de especialistas expertos en los más diversos campos, tales como:

I) Metrología Dimensional;

II) Mediciones Eléctricas y Electrónicas;

III) Acústica ;

IV) Termometría;

V) Fotometría y Radiometría;

VI) Luminotecnia;

VII) Química Industrial;

VIII) Alimentos;

IX) Metalurgia ;

X) Electroquímica;

XI) Ensayos Mecánicos;

XII) Construcción.

Dado este contenido, el INTI se puede comparar con la parte del Consejo Superior de Investigaciones Científicas de España dedicada a la ciencia y a la tecnología: División de Ciencias y Patronato "Juan de la Cierva". En efecto, dentro del INTI, y a través de las amplias y detalladas explicaciones del personal más calificado del complejo, dadas al Prof. CALLEJA, éste pudo identificar, en distintas dependencias del Parque Tecnológico MIGUELETE, cometidos y tareas asignables, en todo o en parte, a bastantes de los diversos Centros e Institutos del Patronato "Juan de la Cierva" del C.S.I.C. español: Instituto de Plásticos y Caucho, Instituto de Investigaciones Pesqueras, Centro de Investigaciones Físicas "Leonardo Torres Quevedo", Centro Nacional de Investigaciones Metalúrgicas, Instituto "Eduardo Torroja" de la Construcción y del Cemento, Instituto de Agroquímica y Tecnología de los Alimentos, Instituto de Optica "Daza de Valdes", Instituto de Tecnología Química y Textil, Instituto de Productos Lácteos, Centro de Investigaciones del Agua, etc.

Asimismo consta el INTI de una Biblioteca Técnica y de servicios bibliográficos especializados, cuyo conjunto es comparable al Centro de Información y Documentación del Patronato "Juan de la Cierva".

El INTI lleva a cabo su misión a través de sus Centros en Buenos Aires, La Plata, Córdoba, Mendoza, San Juan y Rosario de Santa Fe.

Otras dependencias del INTI se asemejan más por la materia de que tratan y por su cometido, a Institutos de la División de Ciencias del C.S.I.C.

De entre lo visto en la detenida visita del Prof. CALLEJA, que ocupó toda una mañana, destacó tal vez lo relativo a Metrología en general, y dentro de ella lo concerniente a calibración y tarado de patrones secundarios y equipos tecnológicos de medición y ensayo, tanto de laboratorio como industriales, en los campos dimensional, eléctrico y electrónico, a.cústico y fotoluminiscente. 


\subsubsection{Coloquio sobre Metrología}

Antes de comenzar la visita propiamente dicha, se celebró un coloquio presidido por el Prof. STEINBERG, figura muy destacada del INTI, a cuyo cargo está toda la parte metrológica del Instituto. Comenzó haciendo una descripción general de los problemas metrológicos, de la actividad del Instituto para resolverlos, de los medios con que éste dispone y líneas de actuación que sigue para desempeñar tal actividad.

Después de responder a algunas preguntas en relación con el tema desarrollado, las cuales le fueron formuladas por el Prof. CALLEJA, éste hizo una breve exposición de la metrología que se ha llevado y lleva a cabo en el IETCC, en relación con el aspecto específico de la construcción, de sus materiales y del control de calidad e idoneidad de los mismos. Hizo mención, en este sentido, de la labor iniciada y desarrollada con todo éxito y promesas por la figura insustituible de su colega Prof. Dr. J. M. TOBIO, prematura y recientemente fallecido.

Finalmente los Profs. STEINBERG y CALLEJA trataron de una posible colaboración hispano-argentina en materias de metrología, dentro de los organismos internacionales especializados en el tema.

En el curso de esta visita el Prof. CALLEJA tuvo ocasión de comprobar el conocimiento y estima que en el INTI se tienen, por parte de su personal más destacado, de miembros del C.S.I.C. español, en relación con el campo de la metrología en los dominios de la óptica, de la acústica y de otras ramas científico-tecnológicas.

\section{CONCLUSION}

Al dar por finalizada la exposición de la reciente actividad científico-técnica española en Iberoamérica, con especial participación del IETCC en ella, el expositor desea hacer constar su más íntima satisfación por la circunstancia de haber sido, en muy gran parte, protagonista de la misma. Y, por haber hecho esto posible, quiere manifestar público agradecimiento, en nombre del IETCC y en el suyo propio, a las entidades y personalidades brasileñas y argentinas por la invitación personal de que le hicieron objeto, y por los múltiples agasajos y atenciones con que le obsequiaron, haciendo alarde de una cordial y generosa hospitalidad. A todos ellos, muchas gracias y hasta siempre. 